\title{
Performance Comparison of IEEE802.11ac vs IEEE 802.11n WLAN in IPv6
}

\author{
Samad S. Kolahi, Ahmad Khalid Sooran, Faroq Nasim, Muhammad Mazhar U. Khan \\ Unitec Unstitute of Technology, Auckland, New Zealand skolahi@unitec.ac.nz \\ khalid-sooran@outlook.com
}

\begin{abstract}
In this paper, performance of WLAN (Wireless LAN) for peer-peer and client-server networks with WPA-2 security is investigated for both IEEE802.11ac and IEEE802.11n standard. Parameters studied include throughput, and RTT (round trip time) of IPv4 and IPv6 protocols for both TCP and UDP. IPv4 outperform IPv6 in terms of bandwidth and delay due its lower overhead size. IEEE $802.11 \mathrm{ac}$ had much better performance than IEEE 802.11 n
\end{abstract}

\section{Introduction}

Wireless connectivity and Wi-Fi are terms most people know about today, and is a service most of us take for granted, in that how important it is and how much easier it has made our daily tasks. From our computers, mobile devices, video game consoles, wireless connectivity allows us to communicate with each other, share resources, and browse the world wide web, all without needing to use cluttered wires, and having the flexibility to freely move around is a bonus. With Wi-Fi, we can connect our devices to an access point and then connect to IoT network, through the use of the 802.11 wireless standards which have helped to bring this medium to homes and businesses all around the world.

This paper aims to present real life performance comparison of the two standards, and identify the significant speed advantage IEEE802.11ac offers over IEEE802.11n in IPv6 environment, for both peer-peer and client networks. The operating system used is Windows 10 and Windows Server 2016. Collected data includes throughout, and round trip time, for TCP (Transmission Control Protocol), UDP (User Datagram Protocol), IPv4 and IPv6. Such data was collected with the aid of a traffic management and data generation tool.

With the many advantages that wireless networking offers, one main disadvantage is being less secure than wired networks, due to devices in a wireless local area network being connected wirelessly over the air, and with this, wireless networks are also slower as there is no direct connection between devices [1].

IEEE802.11n was developed in 2009 to replace IEEE802.11g, offering significantly higher throughput than IEEE802.11g standard. In addition, it has also doubled the connection distance from $450 \mathrm{ft}$. to $820 \mathrm{ft}$. Until recently, it was the most widely used standard in modern times, and is still adequate in supplying networks 
with reliable speeds and security. This standard allows connectivity in the 20 and 40 $\mathrm{MHz}$ channel sizes in addition to offering dual band connection, meaning it can operate in both the $2.4 \mathrm{GHz}$ and $5 \mathrm{GHz}$ bands [2]. To produce higher throughput than its predecessors, this standard is the first to introduce MIMO (multiple input, multiple output) system so that signal strength is boosted, and connections between a client and wireless access point become stronger, leading to better performance. Before the development of IEEE802.11n, a single transmitter and receiver was used to transmit signals between devices in a wireless network, known as a radio chain or SISO (single in, single out) systems [3]. It will also allow multiple channels to be aggregated into a single stream, for example allowing two channels of $20 \mathrm{MHz}$ to be aggregated into a single $40 \mathrm{MHz}$ channel, theoretically doubling throughput [2].

Released commercially in 2013, IEEE802.11ac is the latest wireless standard used in home and business networks around the world, and significantly increase wireless performance both theoretically and practically. Basically, IEEE802.11ac is a super charged version of IEEE802.11n and takes the best features and improves upon them further so that a maximum theoretical throughput of $1.7 \mathrm{Gbps}$ is possible. This is the first wireless standard to take throughput up to gigabit speeds [3]. Unlike IEEE802.11n, IEEE802.11ac only operates at the $5 \mathrm{GHz}$ band and can use 40, 80 and $160 \mathrm{MHz}$ bandwidth sizes, whereas IEEE802.11n only supports 20 and $40 \mathrm{MHz}$ channel sizes. It also uses enhanced MIMO, OFDM (Orthogonal Frequency Division Multiplexing) and beam forming technology to provide higher data rate [2].

IPv6 is the latest version of the Internet Protocol (IP), expected to replace IPv4 in the future and addition to providing backwards compatibility, no need for DHCP server, built in encryption, more efficient routing, elimination of NAT (network address translation), better mobility support, better QoS (Quality of Service) and will address IPv4 address shortage [4].

Some related works regarding performance of IPv6 and WLAN IEEE802.11n and $11 \mathrm{ac}$ are in [5-12].

To the authors knowledge, there is no research to date in the literature on studying performance comparison of IEEE802.11n peer-peer or client-server networks comparing them to IEEE802.11ac. The motivation behind this study therefore is to produce new results to see how much better the new 802.11 ac protocol is, for IPv4, IPv6, TCP, UDP, and for both peer-peer and client server networks.

\section{Network Setup}

To measure the wireless performances, two networks were set up, a peer-peer and a client server network.

For the client-server network, a Windows Server machine was connected to the WAP4410N IEEE802.11n business

wireless access point via a Cat 6 crossover cable for IEEE802.11n testing, and Ubiquiti UniFi HD IEEE802.11ac enterprise access point for IEEE802.11ac testing. The Windows 10 Client machine was connected to the server through the access points wirelessly. The distance between the access points and the client machine was well within few meters, to maintain the maximum signal strength, so that practical 
results obtained are reliable and consistent. The channel bandwidth of the WAP4410N access point was tested using $40 \mathrm{MHz}$, and Ubiquiti UniFi HD $11 \mathrm{ac}$ access point using $80 \mathrm{MHz}$. The hardware specifications for both client and server machines consist of an Intel Core i7 Duo $63002.87 \mathrm{GHz}$, a Western Digital Caviar 160 GB hard drive, 16.00 GB or RAM. The client machine was installed with a TPLink TL-WN951N wireless N network interface card and Asus AC-68 IEEE802.11ac wireless network interface card. The test bed setup remained consistent for all testing conducted in the client-server section. The test bed diagram is shown in Figure 1.

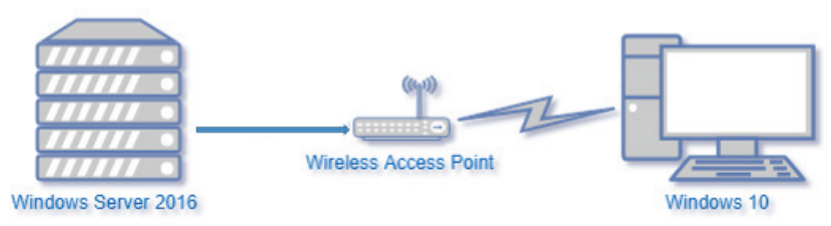

Fig. 1. Client-server test bed

For the peer-peer network, two Windows 10 operated machines were connected to the WAP4410N IEEE802.11n business wireless access point for IEEE802.11n testing, and Ubiquiti UniFi HD for IEEE802.11ac testing wirelessly and a workgroup created to allow them to share resources and communicate with each other. The distance between the access point and the clients were well within few meter, to maintain the maximum signal strength, so that practical results obtained are reliable and consistent. The channel bandwidth for WAP4410N $11 \mathrm{n}$ access point was $40 \mathrm{MHz}$, and $80 \mathrm{MHz}$ channel used for the Ubiquiti IEEE802.11ac access point. The hardware specifications for both client machines consist of an Intel Core i7 Duo 63002.87 GHz, a Western Digital Caviar 160 GB hard drive, 16.00 GB of RAM. The client machines were installed with a TP-Link TL-WN951N wireless N network interface card and Asus AC-68 IEEE802.11ac wireless network interface card. The test bed setup remained consistent for all testing conducted in the client-server section. The test bed diagram is shown in Figure 2.

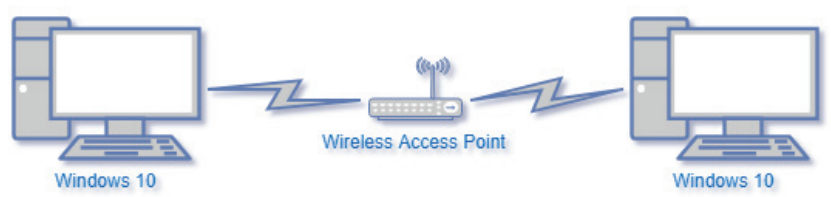

Fig. 2. Peer-peer test bed

\section{Data Generation \& Traffic Measurement Tools}

D-ITG 2.8.1 [13] was the tool used to generate traffic and measure the throughput, and RTT (Round Trip Time). This tool is widely used to evaluate the network performance. 


\section{Practical Results}

This section presents data on the throughput, and RTT of TCP and UDP for both IPv4 and IPv6 on an IEEE802.11 ac and IEEE802.11n peer-peer and client server networks. IEEE802.11ac will be compared to IEEE802.11n. Both networks are set up with WPA-2 security implementation for all the networks. For each packet size, a total of 15 runs are carried out, and the results averaged out with the standard deviation calculated. The standard deviation over the average results of 15 runs was less than $0.5 \%$. As there is a very significant gap in performance between IEEE802.11ac and IEEE802.11n, results will not be directly compared in a single graph, so results are presented in separate graphs for each IEEE standard.

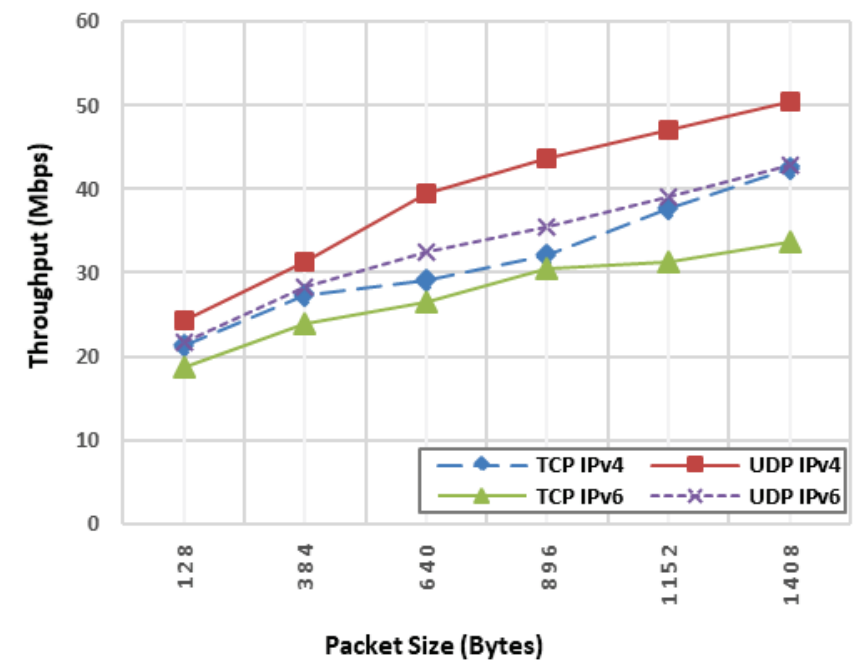

Fig. 3. 802.11n UDP and TCP Throughput Peer-Peer, Wireless Channel Width $40 \mathrm{MHz}$

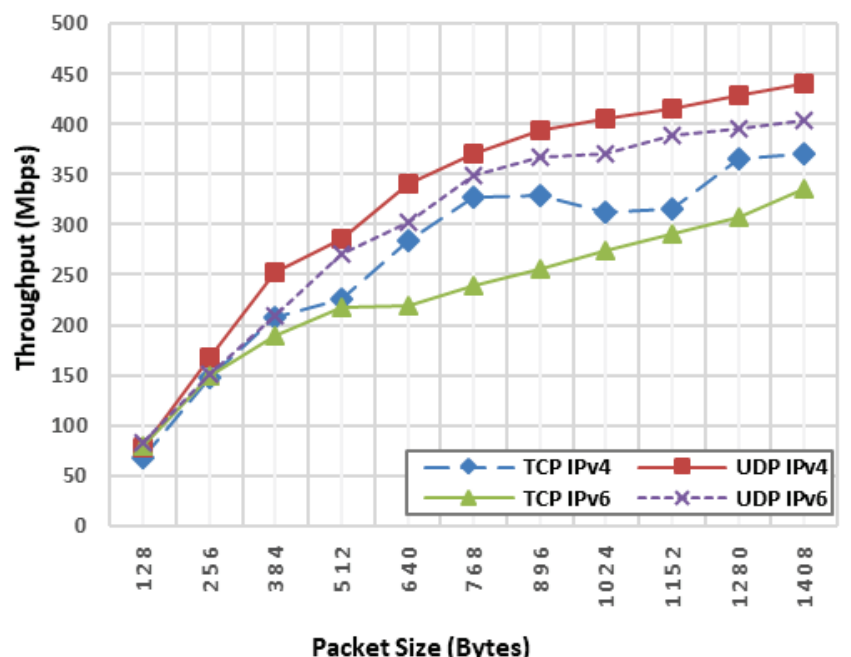

Fig. 4. 802.11ac UDP and TCP Throughput Peer-Peer, Wireless Channel Width 80MHz 
Figure 3 presents throughput data on $802.11 \mathrm{n}$ and Figure 4 presents throughput data on 802.11 ac on peer-peer WLAN networks. Both wireless networks are tested using TCP and UDP protocols for both IPv4 and IPv6. In most scenarios as the packet size increases, TCP and UDP throughout consistently increases alongside the packets. The throughput on $11 \mathrm{ac}$ is significantly greater in all packet sizes tested for both TCP and UDP.

IEEE802.11ac IPv4 UDP outperforms every other packet sizes achieving the highest throughput for all packets except packet 128 Bytes, reaching a maximum throughput of 440 Mbps for packet size of 1408 Bytes. The maximum difference in throughput between $11 \mathrm{n}$ and $1 \mathrm{ac}$ is at packet 1408 Byte for UDP IPv4 802.11ac having 440Mbps and UDP IPv4 802.11n having $50 \mathrm{Mbps}$, where $802.11 \mathrm{ac}$ has an advantage of $390 \mathrm{Mbps}$. For both $802.11 \mathrm{n}$ and 802.11ac, IPv4 TCP and UDP outperform IPv6 TCP and UDP, respectively. This is because IPv6 packet has higher overhead than IPv4, and overheads need processing and that makes IPv6 slower.

Both networks have UDP IPv4 as the highest performing protocol, followed by IPv6 UDP, IPv4 TCP, and then the least performing is IPv6 TCP in both networks. In all scenarios throughput gradually increase as packet size gets higher, except for IPv4 TCP, which drops in speed in packet 896 Bytes to 329Mbps, going down to 313Mbps in packet 1024 Bytes, and then in packet 1152 Bytes increasing again at $316 \mathrm{Mbps}$, this is the only case where throughput had decreased in all scenarios. This was also observed in other research work that graphs are not smooth and could rise and fall a bit as packet size increase [5,7,9], but the overall trend is increasing throughput with packet size. The lowest throughput for $802.11 \mathrm{n}$ was $18 \mathrm{Mbps}$ (at packet 128 Byte) for TCP IPv6, while 802.11ac lowest throughput was 68Mbps (at packet 128 Byte), a significant increase compared to $802.11 \mathrm{n}$.

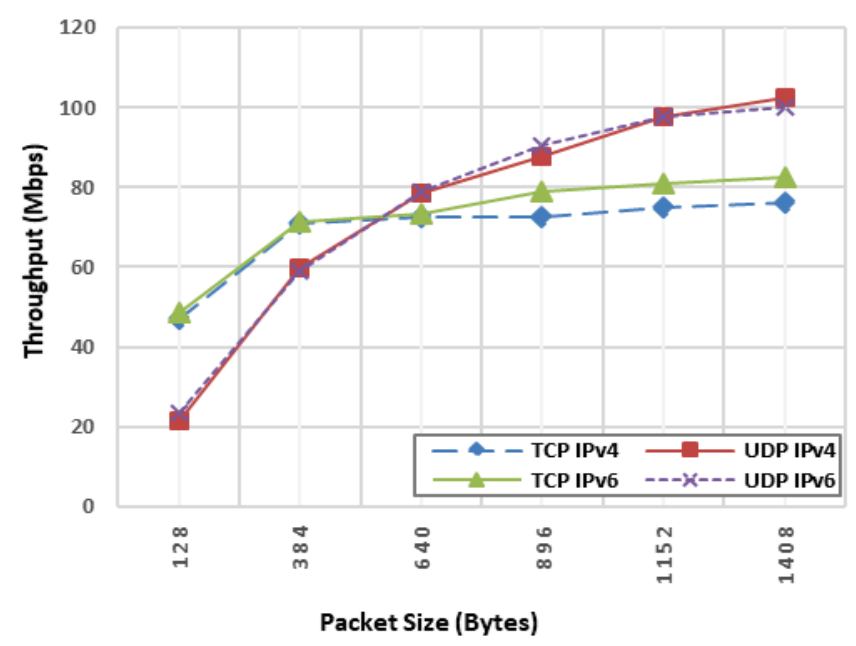

Fig. 5. 802.11n UDP and TCP Throughput Client-Server, Wireless Channel Width 40MHz. 


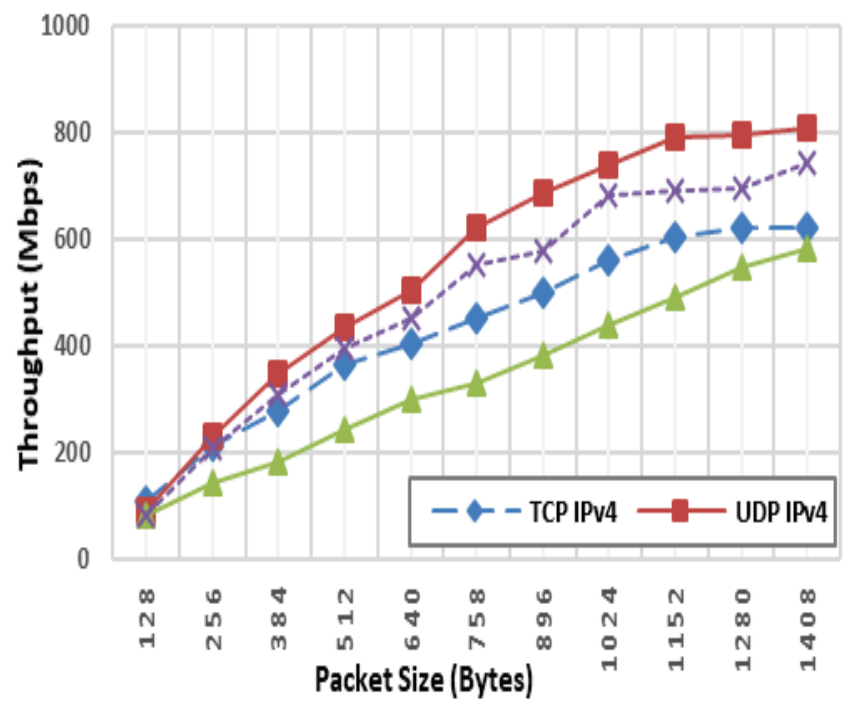

Fig. 6. 802.11ac UDP and TCP Throughput Client-Server, Channel Width 80MHz.

Figure 5 presents throughput data on 802.11 n and Figure 6 is throughput data on 802.11ac on client-server WLAN networks. Both networks are tested using TCP and UDP protocols for both IPv4 and IPv6. 802.11ac using IPv4 and UDP gave the highest throughput of $808 \mathrm{Mbps}$. When comparing $802.11 \mathrm{ac}$ and $802.11 \mathrm{n}$, the maximum difference in throughput difference is for packet 1408 Byte, UDP IPv4 802.11ac having $808 \mathrm{Mbps}$ and IPv4 UDP 802.11n having 102Mbps, which makes a huge difference of $706 \mathrm{Mbps}$, showing just how fast $802.11 \mathrm{ac}$ is over its predecessor. IPv4 outperforms IPv6 for TCP and UDP as it has less overhead than IPv6. Both networks have UDP IPv4 as the highest performing protocol, followed by IPv6 UDP, IPv4 TCP, and then the least performing is IPv6 TCP in both networks. One point of deviation is at Figure $5(802.11 \mathrm{n})$ where TCP outperforms UDP on packets 128 and 384 for both IPv4 and IPv6. At low packet sizes, the data obtained had some randomness and this was observed in other studies too $[5,7,9]$.

TCP has lower throughput as it sends acknowledgements, and that it has higher overhead in its packet. Generally, both networks have UDP IPv4 as the highest performing protocol, followed by IPv6 UDP, IPv4 TCP, and then the least performing is IPv6 TCP in both networks. The lowest throughput is in $802.11 \mathrm{n}$ at packet size 128Byte for UDP IPv6 achieving 20Mbps, while 802.11ac TCP IPv4 at packet $128 \mathrm{Byte}$ achieve $105 \mathrm{Mbps}$, which is a significant increase. 


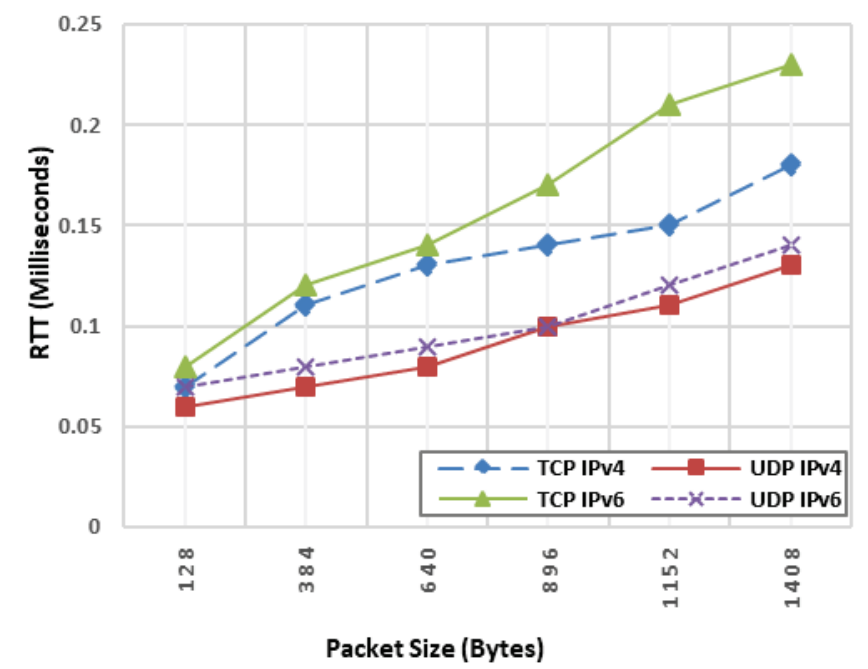

Fig. 7. 802.11n UDP and TCP RTT Peer-Peer, Channel Width 40MHz.

Figure 7 presents the RTT (round trip time) of the 802.11n peer-peer WLAN network. UDP IPv4 had the least round trip time for each packet, steadily rising as the packet size rises. It achieved a round trip time of 0.07 milliseconds at packet 128 Bytes, while having 0.18 milliseconds delay at packet 1408 Bytes. The slowest is TCP IPv6, with 0.23 milliseconds at packet 1408 Bytes. Overall, UDP outperforms TCP for both IPv4 and IPv6. As discussed earlier. The reason for above is that because UDP packet has less overhead than TCP, TCP sends acknowledgements being a connection oriented protocol, and that IPv4 packet has less overhead than IPv6.

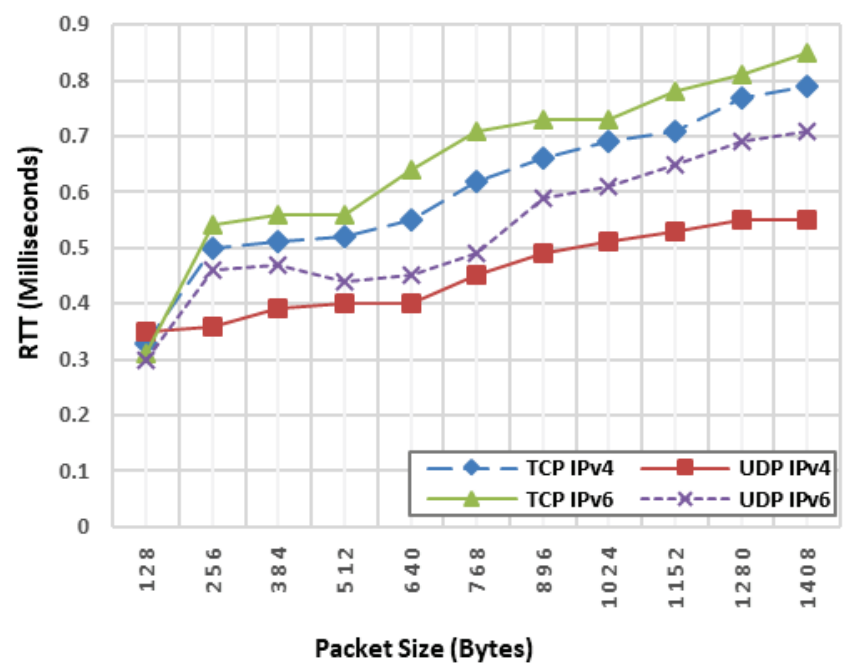

Fig. 8. 802.11ac UDP and TCP RTT Peer-Peer Channel Width 80MHz. 
Figure 8 presents the RTT of the 802.11 ac peer-peer WLAN network. IPv4 UDP achieved lower RTT than all other scenarios. It achieved a round trip time of 0.35 milliseconds at packet 128 Byte, while having 0.5 milliseconds at packet 1408 Byte. The highest RTT was for TCP IPv6, with 0.85 milliseconds at packet 1408 Byte. Overall, UDP outperforms TCP for both IPv4 and IPv6. Both networks have UDP IPv4 as the best performing protocol, and having IPv6 TCP as the protocol with the highest latency, due to the IPv6 TCP header being larger than all of them. All protocols had gradually increased in latency as packet sizes increased, however only UDP IPv6 had a drop in packet 512 Bytes going to 0.44 milliseconds, while at packet size 384 Bytes had achieved 0.47 milliseconds RTT. After this packet, latency gradually increases again, with packet size 640 Bytes having 0.45 milliseconds, going to 0.50 milliseconds for packet size 768 Bytes.

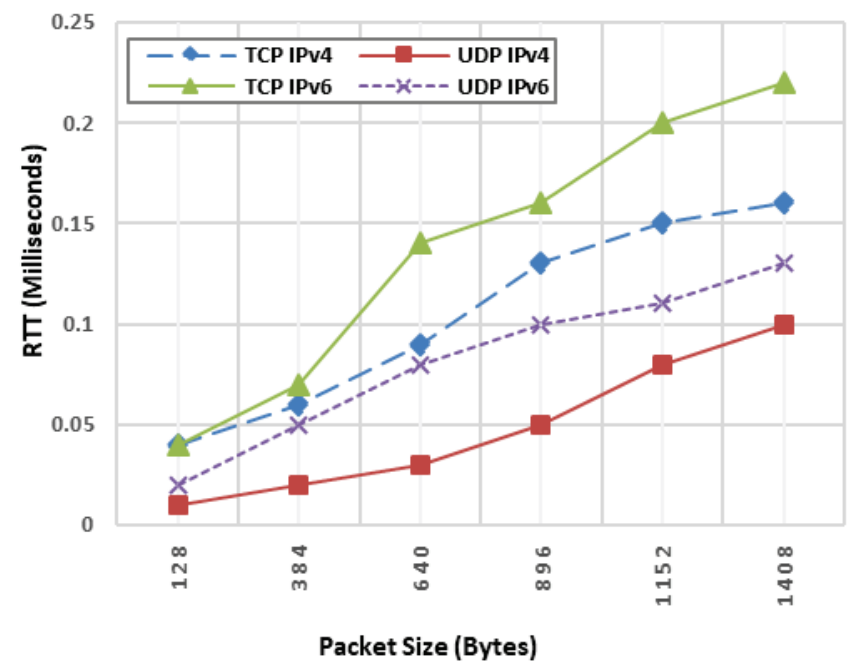

Fig. 9. 802.11n UDP and TCP RTT Client-Server, Channel Width 40MHz.

Figure 9 presents the RTT of the 802.11n client-server WLAN network. UDP IPv4 had the least round trip time for each packet, steadily rising as the packet rises. It achieved a round trip time of 0.01 milliseconds at packet 128 Bytes, this could be because the client-server connection through a wire is much better than that of peerpeer wireless connection over the air. It had the latency of 0.13 milliseconds at packet 1408 Bytes. The slowest is TCP IPv6, taking 0.22 milliseconds at packet 1408 Bytes. Overall client server had better latency than peer-peer counterpart. UDP outperforms TCP for both IPv4 and IPv6. IPv4 have better RTT performance than IPv6. Note these numbers are quite low ranging in the milliseconds, so performance in the real world would be hardly noticeable, and would basically be the same. 


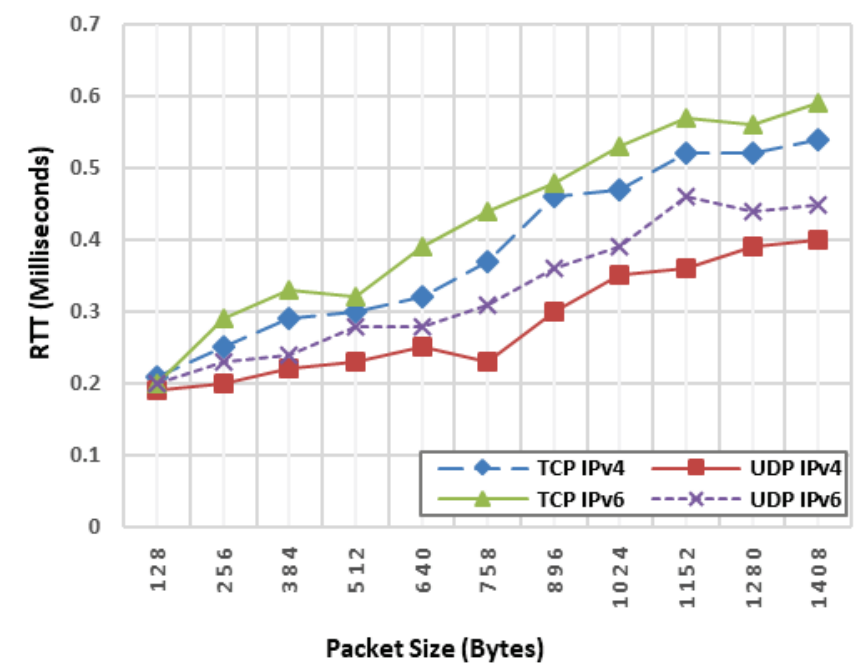

Fig. 10. 802.11ac UDP and TCP RTT Client-Server, Channel Width 80MHz.

Figure 10 is the RTT data as collected from 802.11ac client-server network. UDP IPv4 having 0.19 seconds round trip time at packet 128Bytes, while achieving 0.4 milliseconds at packet 1408 Bytes. This delay is significantly better than peer-peer wireless network, due to a server connected to the access point through a wire. TCP IPv6, as expected, had the highest latency, due to the large header in IPv6 and higher overhead in TCP, and connection oriented nature of TCP.

\section{Conclusions}

In this paper, due to the advantages and features of IEEE802.11ac over IEEE802.11n, it gave the highest throughput for both peer-peer and client-server networks at 444Mbps (IPv4 UDP) and 808Mbps (IPv4 UDP), respectively. This throughput is lower than the 1.7 Gbps theoretical bandwidth of 802.11ac. Peer-peer network bandwidth was much less than client server. UDP performed better (higher bandwidth) than TCP, and IPv4 provided higher bandwidth than IPv6. The comparison results from above protocols was presented in the paper.

\section{Future Work}

The future work includes testing more operating systems such as Linux, using multiple client machines for multi machine throughput testing, and also open system testing to compare with WPA2 encryption results of this study. 


\section{References}

1. Zeng, Y., Pathak, Mohapatra, P.: Throughput, energy efficiency and interference characterisation of $802.11 \mathrm{ac}$, Transactions on Emerging Telecommunications Technologies, 2015.

2. CISCO. CCNA Wireless - Study Notes Part 2: 802.11 standards and WLAN Terminology. Learning Network Cisco: https://learningnetwork.cisco.com/docs/DOC-31396, 2016

3. Bradley, M., What Is Multiple-In Multiple-Out (MIMO) Technology?:

https://www.lifewire.com/mimo-wifi-routers-818332

4. Microsoft, "Explore Windows 10 New Features and Update". Retrieved from Microsoft: https://www.microsoft.com/en-nz/windows/features, April 2018.

5. Kolahi, S.S., Almatrook, A. A.: Impact of security on bandwidth and latency in IEEE IEEE802.11 ac client-to-server WLAN. 2017 Ninth International Conference on Ubiquitous and Future Networks (ICUFN), pp. 893-897, 2017.

6. Newell. P.: Comparison of Theoretical and Practical Performances with $802.11 \mathrm{n}$ and 802.11ac Wireless Networking. 2017 31st International Conference on Advanced Information Networking and Applications Workshops (WAINA), pp. 710-715, 2017.

7. Kolahi, S. S. Cao, Y., Chen, H. : Impact of SSL security on bandwidth and delay in IEEE IEEE802.11n WLAN using Windows 7. 2016 10th International Symposium on Communication Systems, Networks and Digital Signal Processing (CSNDSP), 2016.

8. Shah, Z.: Throughput Comparison of IEEE 802.11 ac and 802.11n in an Indoor Environment with Interference. 2015 International Telecommunication Networks and Applications Conference (ITNAC), pp. 196-201, 2015.

9. Kolahi, S. S., Cao, Y.R., Chen H.: Bandwidth-IPSec security trade-off in IPv4 and IPv6 in Windows 7 environment, Second International Conference on Future Generation Communication Technologies (FGCT), 2013.

10. Dianu, M. D., Riihijarvi, J., and Petrova. M.: Measurement-based study of the performance of IEEE 802.11ac in an indoor environment, IEEE International Conference on Communications (ICC'14), Sydney, pp. 5771 - 5776, 2014.

11. Park. M.: IEEE 802.11ac: Dynamic bandwidth channel access,, IEEE International Conference on Communications (ICC'11), Kyoto, Japan, 5-9 June 2011, pp. 1 - 5.

12. Siddiqui, F., Zeadally, S., and Salah, K.: Gigabit Wireless Networking with IEEE 802.11ac: technical overview and challenges, Journal of Networks, vol. 10, no. 3, pp. 164 - 171, Apr. 2015.

13, GRID. (n.d.). D-ITG, Distributed Internet Traffic Generator. Retrieved March 28, 2018, from GRID: http://www.grid.unina.it/software/ITG/. 